\title{
Comparative analyses of angiosperm secretomes identify apoplastic pollen tube functions and novel secreted peptides
}

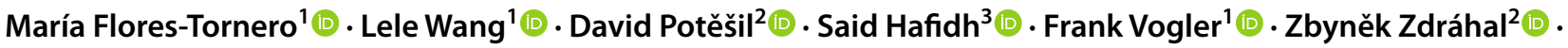 \\ David Honys $^{3}$ (D) Stefanie Sprunck ${ }^{1}$. . Thomas Dresselhaus $^{1}[$
}

Received: 23 September 2020 / Accepted: 10 November 2020 / Published online: 30 November 2020

(c) The Author(s) 2020

\begin{abstract}
Key message Analyses of secretomes of in vitro grown pollen tubes from Amborella, maize and tobacco identified many components of processes associated with the cell wall, signaling and metabolism as well as novel small secreted peptides.

Abstract Flowering plants (angiosperms) generate pollen grains that germinate on the stigma and produce tubes to transport their sperm cells cargo deep into the maternal reproductive tissues toward the ovules for a double fertilization process. During their journey, pollen tubes secrete many proteins (secreted proteome or secretome) required, for example, for communication with the maternal reproductive tissues, to build a solid own cell wall that withstands their high turgor pressure while softening simultaneously maternal cell wall tissue. The composition and species specificity or family specificity of the pollen tube secretome is poorly understood. Here, we provide a suitable method to obtain the pollen tube secretome from in vitro grown pollen tubes of the basal angiosperm Amborella trichopoda (Amborella) and the Poaceae model maize. The previously published secretome of tobacco pollen tubes was used as an example of eudicotyledonous plants in this comparative study. The secretome of the three species is each strongly different compared to the respective protein composition of pollen grains and tubes. In Amborella and maize, about 40\% proteins are secreted by the conventional "classic" pathway and 30\% by unconventional pathways. The latter pathway is expanded in tobacco. Proteins enriched in the secretome are especially involved in functions associated with the cell wall, cell surface, energy and lipid metabolism, proteolysis and redox processes. Expansins, pectin methylesterase inhibitors and RALFs are enriched in maize, while tobacco secretes many proteins involved, for example, in proteolysis and signaling. While the majority of proteins detected in the secretome occur also in pollen grains and pollen tubes, and correlate in the number of mapped peptides with relative gene expression levels, some novel secreted small proteins were identified. Moreover, the identification of secreted proteins containing pro-peptides indicates that these are processed in the apoplast. In conclusion, we provide a proteome resource from three distinct angiosperm clades that can be utilized among others to study the localization, abundance and processing of known secreted proteins and help to identify novel pollen tube secreted proteins for functional studies.
\end{abstract}

Keywords Pollen tube $\cdot$ Proteomics $\cdot$ Secretome $\cdot$ Cell wall $\cdot$ CRP $\cdot$ Signaling $\cdot$ Amborella $\cdot$ Maize $\cdot$ Tobacco

\section{Introduction}

Communicated by Dolf Weijers.

Electronic supplementary material The online version of this article (https://doi.org/10.1007/s00497-020-00399-5) contains supplementary material, which is available to authorized users.

Thomas Dresselhaus

thomas.dresselhaus@ur.de

Extended author information available on the last page of the article
In flowering plants (angiosperms), sperm cells have lost their mobility and are transported as a passive cargo by pollen tubes deep into the maternal tissues of the ovary toward ovules (Zhang et al. 2017). Inside ovules, sperm cells are released for a double fertilization process to generate an embryo and endosperm, respectively (Dresselhaus et al. 2016). During their journey, pollen tubes communicate intensively with female reproductive tissues starting with papilla cells of the stigma during germination, transmitting 
tract cells during further growth, maternal cells of the ovule as well as cells of the female gametophyte (embryo sac) for guidance and attraction culminating in pollen tube burst (Dresselhaus and Franklin-Tong 2013; Johnson et al. 2019; Zhou and Dresselhaus 2019). Interaction with the synergid cells, secretory cells of the embryo sac, is especially critical as these regulate attraction during the last passage of the journey and termination during sperm cell perception (Maruyama and Higashiyama 2016).

During their journey, pollen tubes therefore secrete many different types of proteins and peptides (secretome) (Johnson et al. 2019; Qu et al. 2015). The secretome is generally considered as the sum of proteins secreted into the extracellular space of a plant cell or tissue at any given time and under certain conditions through various secretory mechanisms (Agrawal et al. 2010). Although it is difficult to identify secretome components in vivo, genetic and transcriptomic studies have uncovered that pollen tubes secrete various types of signaling ligands including chemocyanins and plantacyanins (Chae and Lord 2011) as well as small cysteine-rich proteins (CRPs) like LAT52 (Muschietti et al. 1994), rapid alkalinization factors (RALFs) (Ge et al. 2017; Mecchia et al. 2017) and lipid transfer proteins (LTPs) (Chae et al. 2010). The roles of some secreted peptides/proteins in pollen tube growth, guidance or cell wall integrity have been elucidated. CRPs appear especially important as they are overrepresented in pollen tubes compared with vegetative tissues (Bircheneder and Dresselhaus 2016; Huang et al. 2015). Additionally, many secreted proteins are involved in formation, modification and remodeling of the pollen tube cell wall, such as expansins, pectin methylesterases (PMEs) and their inhibitors (PMEIs), pectin lyases and glycoside hydrolases (Dehors et al. 2019; Mollet et al. 2013).

To reach the extracellular space, it was initially thought that most proteins secreted from pollen tubes contain a $\mathrm{N}$-terminal signal peptide to be targeted and processed through the classical/conventional secretory route via the endoplasmic reticulum, Golgi and trans-Golgi network. However, recent studies have shown that about 50\% secreted plant proteins lack known $N$-terminal signal peptides indicating alternative/unconventional secretion pathways involving, for example, the EXPO complex (Krause et al. 2013). Until now, only few studies support the presence of unconventional secretory pathways also for pollen tube secretions as shown for Olea europaea (olive tree) (Alché et al. 2004; Prado et al. 2014) and Nicotiana tabacum (tobacco) (Hafidh et al. 2016), respectively.

Despite these studies, our current knowledge about the exact composition and function of the protein/peptide components of pollen tube secretomes is far from being complete. In order to elucidate secretome components of pollen tubes from various plant species, we describe here an easy and reproducible protocol that can be widely applied to identify secreted proteins from in vitro grown pollen tubes. The basal angiosperm Amborella trichopoda, the monocotyledonous crop and grass model Zea mays (maize) and the eudicotyledonous plant Nicotiana tabacum (tobacco) have then been used as representatives of different angiosperm clades to compare the components of their respective secretomes, the prevalence of conventional versus unconventional secretory pathways and associated functions of secreted proteins. Finally, we show how this approach can be used to identify novel secreted small peptides and how the secretome can be exploited to elucidate, for example, processing of secreted proteins.

\section{Materials and methods}

\section{Plant material and growth conditions}

Male flowers of Amborella trichopoda (Amborella) were harvested at the Botanical Garden in Bonn (Germany). Plants were grown in a shaded place inside a greenhouse under controlled conditions of $16-18^{\circ} \mathrm{C}$, constant humidity of about $66 \%$ and 12 -h photoperiods. Fully opened male flowers were gathered in 50-ml Falcon ${ }^{\mathrm{TM}}$ conical tubes (Thermo Fisher), placed without lid in a hermetically sealed plastic box containing a bed of silica gel and stored at $4{ }^{\circ} \mathrm{C}$ until pollen isolation. Mature pollen grains from Zea mays (maize) were obtained from B73 inbred line. Seeds were germinated in a humid chamber and transferred 5 days later to individual $10-\mathrm{cm}$-diameter pots with a soil and substrate mixture $(1: 1, \mathrm{v} / \mathrm{v})$. Maize seedlings were then transferred to $10-\mathrm{L}$ pots in the greenhouse under controlled conditions of a constant air humidity of $60-65 \%, 14 \mathrm{~h}$ of light at $26^{\circ} \mathrm{C}$ and $10 \mathrm{~h}$ of darkness at $21^{\circ} \mathrm{C}$. Supplemented light of $16,000 \mathrm{~lx}$ was provided to adjust day length duration. Plants were watered by using an automated temperature-water-based irrigation system and were fertilized twice a week with $2 \%$ Hakaphos (Compo Expert).

\section{Obtainment of pollen tube secretomes}

In order to obtain proteins secreted from growing pollen tubes (secretome), mature pollen grains from Amborella were isolated as described in Flores-Tornero et al. (2020). In brief, male flowers were rehydrated for $30 \mathrm{~min}$ and resuspended in fresh sterile-filtered Amborella pollen germination medium (5\% sucrose, $300 \mu \mathrm{g} / \mathrm{mL} \mathrm{Ca}\left(\mathrm{NO}_{3}\right)_{2}, 200 \mu \mathrm{g} /$ $\mathrm{mL} \mathrm{MgSO}_{4}, 100 \mu \mathrm{g} / \mathrm{mL} \mathrm{KNO}_{3}, 100 \mu \mathrm{g} / \mathrm{mL} \mathrm{H}_{3} \mathrm{BO}_{3}$ and $\mathrm{pH}$ 5.0). After several filtering steps with pluriStrainer® 70-30 and 15-micron pore size strainers, clean pollen was obtained as a beige-white clean film on the 15-micron strainer and placed in a $1.5-\mathrm{mL}$ collection tube. Fifty milligrams of clean pollen was immediately frozen in liquid nitrogen for protein 
extraction and stored at $-80^{\circ} \mathrm{C}$. The rest of the clean pollen was resuspended in pollen germination media, and $1 \mathrm{ml}$ of pollen suspension $\left(\mathrm{OD}_{600}=1\right)$ was diluted to a final volume of $5 \mathrm{~mL}$, poured into $5.5-\mathrm{cm}$-diameter glass Petri dishes and incubated in a humid chamber at room temperature for up to $20 \mathrm{~h}$ on the laboratory bench. Fresh pollen was harvested directly from maize tassels in the morning, and $50 \mathrm{mg}$ pollen was resuspended per $1 \mathrm{~mL}$ maize pollen germination medium ( $15 \%$ sucrose, $0.06 \% \mathrm{Ca}\left(\mathrm{NO}_{3}\right), 0.02 \% \mathrm{MgSO}_{4}$, $0.01 \% \mathrm{KNO}_{3}, \mathrm{pH} 5.0$ ) and incubated for $20 \mathrm{~min}$ at room temperature. After incubation, successful pollen germination and tube formation in Amborella and maize samples was confirmed with a Zeiss Axioscope and a Nikon Eclipse TE2000-S inverted microscope, respectively. The solution containing pollen tubes and its secreted proteins was filtered and pollen tubes were retained by filtration through a 40-micron strainer, whereas the non-germinated pollen grains were retained in the 15-micron strainer (see also Fig. 1a for an overview). Fifty milligrams of pollen tubes was collected from the surface of the 40-micron strainer with a spatula, placed in a $1.5-\mathrm{mL}$ collection tube, immediately frozen in liquid nitrogen for protein extraction and stored at $-80^{\circ} \mathrm{C}$. The $5 \mathrm{~mL}$ flowthrough containing secreted proteins was concentrated at $4{ }^{\circ} \mathrm{C}$ with Amicon ${ }^{\circledR}$ Ultra-centrifugal filter $3 \mathrm{kDa} \mathrm{MWCO}$ according to the manufacturer instructions, aliquoted into $25 \mu \mathrm{L}$ volume and stored at $-80^{\circ} \mathrm{C}$. The total number of independent biological replicates used to extract proteins for pollen grains, pollen tubes and secretome was each 6 for Amborella and 4 for maize.

\section{Protein extraction and LC-MS/MS analysis}

Total proteins from $50 \mathrm{mg}$ of pollen grains and pollen tubes were extracted by grinding samples with liquid nitrogen in a mortar and subsequent addition of $250 \mu \mathrm{L}$ of ice-cold extraction buffer $(50 \mathrm{mM}$ Tris $/ \mathrm{HCl}, 150 \mathrm{mM} \mathrm{NaCl}, 0.1 \%$ sodium deoxycholate, $0.1 \%$ Triton-X100, 1 mM PMSF, pH 8.0). Pollen protein extracts were washed twice in a bench centrifuge

A

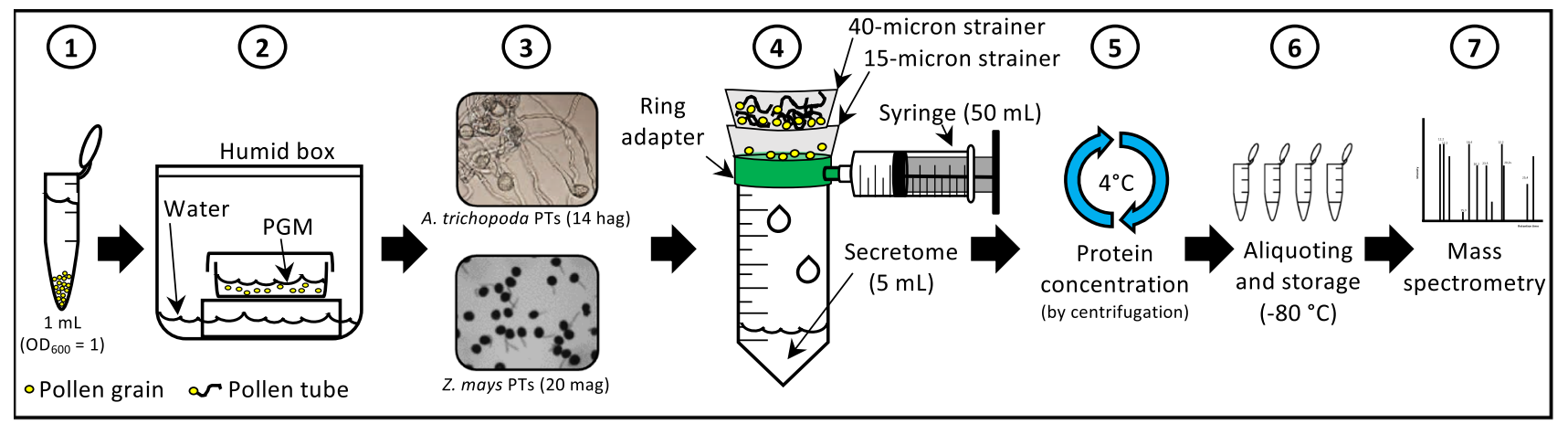

B

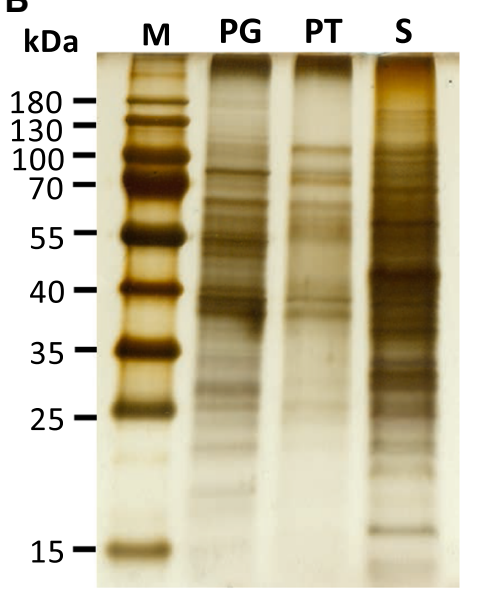

C

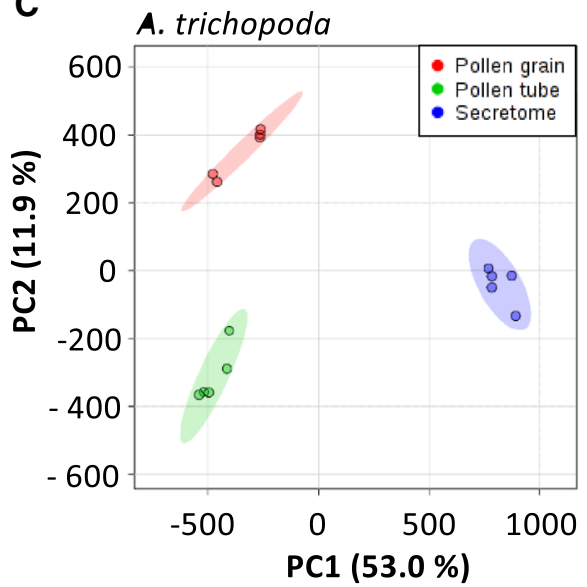

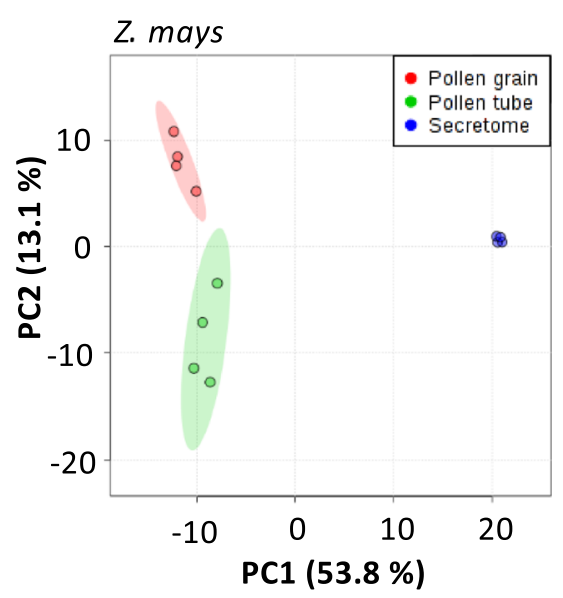

Fig. 1 Procedure to obtain the pollen secretome from Amborella trichopoda and Zea mays and data quality analysis. a Schematic diagram describing the process of secretome obtainment in seven steps as indicated (see text for details). b Representative silver stained SDS-PAGEs showing differences in protein profiles of pollen grains
(PG), pollen tubes (PT) and secretome (S) from A. trichopoda. c Principal component analysis (PCA) of the proteomic profile of $A$. trichopoda and Z. mays. Abbreviations: hag, hours after germination; mag, minutes after germination; PC, principal component; PGM, pollen germination medium 
$\left(13,000 \mathrm{rpm}, 10 \mathrm{~min}, 4^{\circ} \mathrm{C}\right)$, distributed in aliquots of $50 \mu \mathrm{L}$ and stored at $-80^{\circ} \mathrm{C}$. To analyze protein extracts, $10 \mu \mathrm{g}$ of each biological replicate from pollen grains, pollen tubes and secreted proteins were loaded on a $12 \%$ SDS-PAGE using PageRuler ${ }^{\mathrm{TM}}$ Prestained Protein Ladder as marker (Thermo Fischer) and detected by silver staining according to Chevallet et al. (2006). Proteomic analyses were done as described (Hafidh et al. 2016). In short, protein solutions were processed by filter-aided sample preparation (FASP) using the Microcon device with MWCO 30 kDa (Merck Millipore) including alkylation (iodoacetamide, Sigma-Aldrich) and digestion step (trypsin, Promega). Liquid chromatography/mass spectrometric (LC-MS/MS) analyses of peptide mixtures were done using the UltiMate 3000 RSLCnano system connected to an Orbitrap Elite hybrid mass spectrometer (Thermo Fisher Scientific). MS data were acquired in a data-dependent strategy selecting up to top 10 precursors for higher-energy collisional dissociation (HCD) fragmentation. The analysis of mass spectrometric raw data files was carried out using the Proteome Discoverer software (Thermo Fisher Scientific; version 1.4) with in-house Mascot (Matrix Science; version 2.6) and Sequest search engines utilization. MS/MS ion searches were done against the modified cRAP database (based on https://www.thegpm.org/crap/) and UniProtKB protein database for both Amborella trichopoda (https://www.uniprot.org/proteomes/UP000017836; downloaded 18.4.2016, number of protein sequences 27,371) and Zea mays (ftp://ftp.uniprot.org/pub/databases/unipr ot/current_release/knowledgebase/reference_proteomes/ Eukaryota/UP000007305_4577; version 2017-07; 39,441 protein sequences). Percolator was used for post-processing of search results. Peptides with $q$ value $<0.01$, rank 1 and at least 6 amino acids long were considered only. Proteins abundance was assessed using protein area calculated by Proteome Discoverer software (Thermo Fisher Scientific; version 1.4). Peptides were either unambiguously assigned to a single protein record in UniProt database (Accession) or could match several proteins. In this case, the accessions of all these matching candidates were combined into supergroups (SG) and listed according to their match probability. The most probable candidate is identified as "master protein" and is always listed in the first position. Each SG was quantified as maximum of intensities of proteins in a protein group or as $90 \%$ of the minimal observed intensity in a sample. Then, $\log 10$ transformation was applied, and the data were linearly normalized to equalize the median intensities in all samples.

\section{Bioinformatic analyses}

A statistical overrepresentation of gene ontology (GO) terms related to biological process was obtained from PANTHER version 15.0 (https://www.pantherdb.org). The in silico predictions for classical and non-classical secretory pathways were done using the default settings in SecretomeP v2.0, SignalP v5.0 and ProSite (https://prosite.expasy.org). Transcriptomic data from Amborella and maize were obtained from CoNekT online database (https://evorepro. sbs.ntu.edu.sg). The proteomic data from Nicotiana tabacum were obtained from Supplementary Table S4 from the publication Hafidh et al. (2016). We further processed the data and considered only proteins present in all replicates from the tobacco secreted samples (a total of 1375 proteins). As most of these proteins either did not have a valid ID to obtain information from UniProtKB or were obsolete in that database, a manual re-annotation based on the respective amino acidic sequences was performed. Re-annotation was made by bulk blast of amino acidic sequences against the UniProtKB database. By this procedure, only information from 798 tobacco proteins was recovered and used in this comparative study.

\section{Results}

\section{Establishment of a simple and reproducible procedure to obtain the secretome of in vitro grown pollen tubes}

To study the composition and nature of pollen tube secretions (hereafter named as secretome), we first isolated four replicates of fresh pollen from maize and six from Amborella as described (Flores-Tornero et al. 2020). Pollen grains from each replicate were separated for later protein extraction, and the rest were used for obtaining the secretome as described in Fig. 1a. In general, this procedure consisted of resuspending pollen grains in pollen germination medium and incubation in a humid box to induce germination. After $14 \mathrm{~h}$ for Amborella and 20 min for maize, secretions of growing pollen tubes were collected in liquid medium as shown in Fig. 1a. After checking the presence of pollen tubes, pollen germination media containing secreted proteins were filtered to separate pollen tubes and ungerminated pollen grains, respectively. Retained pollen tubes were recovered and used for protein extraction, whereas germination media containing the secretome were further concentrated by subsequent centrifugation, aliquoted and stored at $-80{ }^{\circ} \mathrm{C}$ for LC-MS/ MS analysis.

Silver-stained SDS-PAGE gels showed that protein extractions from pollen grains (PG), pollen tubes (PT) and the secretome (S) of Amborella look very different (Fig. 1b). In maize, PG and PT samples appeared more similar, while the S sample was also strongly different (Supplemental Fig. S1). A principal component analysis (PCA) of the data obtained by LC-MS/MS confirmed this observation and additionally showed that all biological replicates generated 
from the same sample group locate closely together showing the reproducibility of the data generated (Fig. 1c). Moreover, the finding that the secretome data points of both species were very homogenous and very distant from those of pollen grains and pollen tubes, which plot more closer to each other, further indicates that the secretome is not contaminated by burst pollen tubes.

\section{About $70 \%$ proteins detected in the pollen tube secretome are also in silico predicted to be secreted}

In order to analyze the composition of the secretomes generated and to compare it with proteins present in pollen grains and pollen tubes, respectively, samples from Amborella and maize were first analyzed by LC-MS/MS. Complete proteomic reports are provided in Supplemental Table S1 (Amborella) and Supplemental Table S2 (maize), respectively. Additionally, data for the tobacco secretome that has been generated in a previous study (Hafidh et al. 2016) were extracted from the corresponding supplementary material, further processed as described in Materials and Methods and is now provided as Supplemental Table S3 (tobacco). A total of 3658 proteins in all samples were detected in Amborella, 3979 in maize and 9822 in tobacco. For further analysis, we considered as "secreted" only those proteins with at least one peptide detected in at least two biological replicates of the secretome samples. About $12 \%$ of all proteins matched this criterion in all three plant species (Fig. 2a). Totally, 335 proteins were considered to be secreted from Amborella pollen tubes, 552 from maize and 1375 from tobacco pollen tubes. However, in contrast to Amborella and maize, about $42 \%$ proteins detected in the tobacco secretome did not have a valid ID and we thus continued only with the 798 proteins that were registered in UniProtKB and which were clearly associated with tobacco.

The majority of proteins detected in the Amborella samples $(76 \%)$ were initially reported as "uncharacterized." In maize and tobacco, these numbers were much lower $(7.6 \%$ and $3 \%$, respectively). In order to obtain more information about functions of detected proteins, we developed a
A

\section{A. trichopoda (3,658 proteins)}

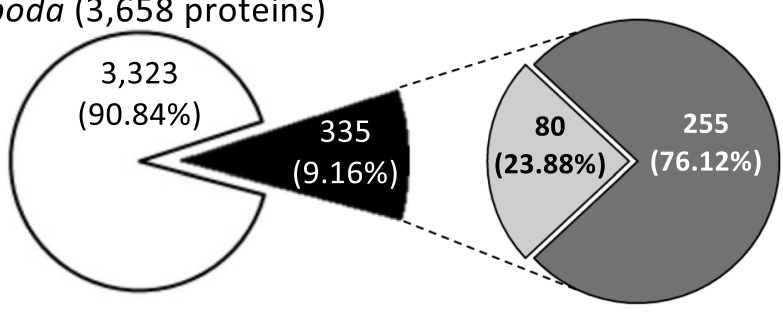

Z. mays (3,979 proteins)

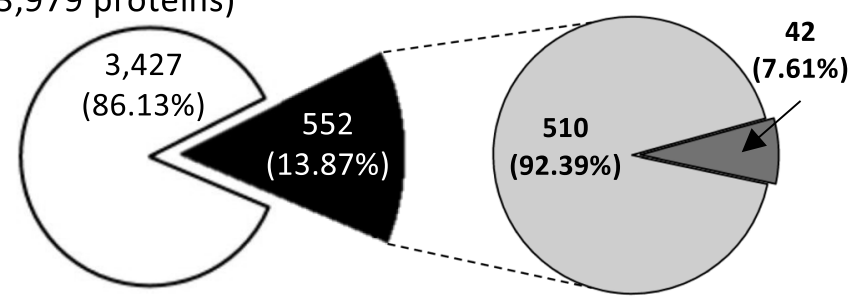

N. tabacum $(9,822$ proteins)

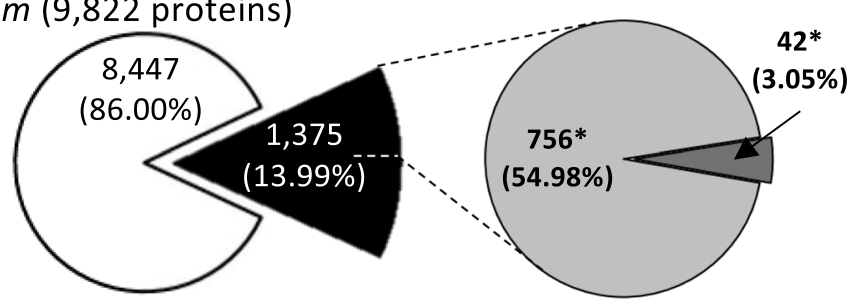

Secreted

\section{Characterized}

Uncharacterized

\section{B}

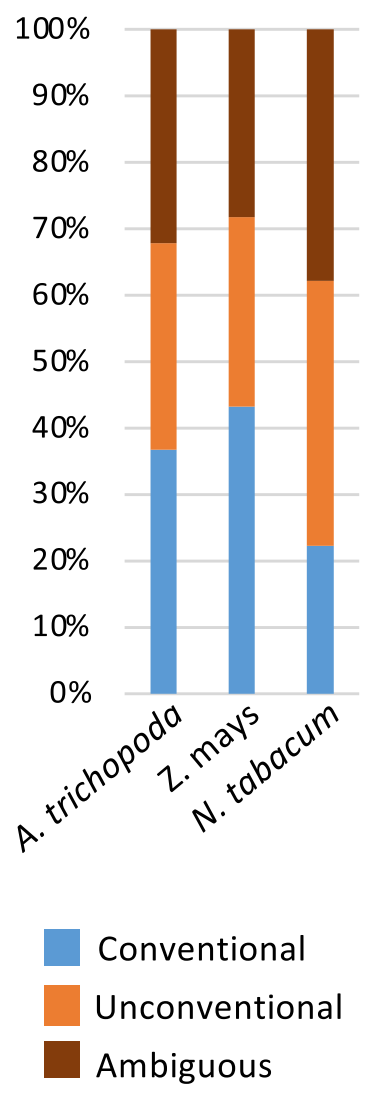

Fig. 2 Statistical analysis of proteomic data obtained from pollen grains, pollen tubes and their secretome of A. trichopoda, $Z$. mays and Nicotiana tabacum. a Total number of proteins classified as "secreted" (detected in at least two biological replicates in the secretome data), "characterized" (described in the proteomic report) or "uncharacterized" (without description in the proteomic report). (*) Number of $N$. tabacum proteins with available UniProt ID (see text for details). b Predicted relative number of secreted proteins for conventional and unconventional secretory pathways, respectively 
A

GO terms

(Biological process)

Z. mays

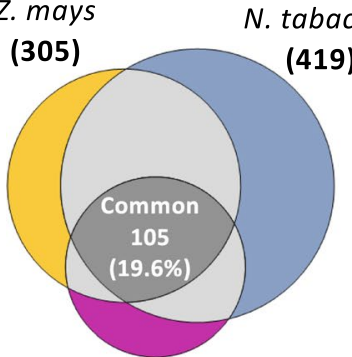

A. trichopoda

(181)
B

\section{GO terms}

GO:0005975_Carbohydrate metabolism GO:0006091_Generation of metabolites and energy GO:0006098_Pentose-phosphate shunt GO:0006082 Organic acid metabolic process GO:0006090_Pyruvate metabolic process GO:0006099 Tricarboxylic acid cycle GO:0032787_Monocarboxylic acid metabolic process GO:0006457 Protein folding

GO:0046939_Nucleotide phosphorylation GO:0072521_Purine metabolic process GO:1901565_Organonitrogen compound catabolism GO:0042744_Hydrogen peroxide catabolic process GO:0055114_Oxidation-reduction process GO:0072593_Reactive oxygen species metabolism GO:0019222_Regulation of metabolic process GO:0043086_Negative regulation of catalytic activity GO:0044281_Small molecule metabolic process GO:0019722_Calcium-mediated signaling GO:0051645_Golgi localization

GO:0071554_Cell wall organization or biogenesis GO:0006979_Response to oxidative stress

GO:0010035_Response to inorganic substance GO:0034620_Cellular response to unfolded protein GO:0035966_Response to topologically incorrect protein GO:0042221_Response to chemical GO:0097237_Cellular response to toxic substance GO:0098869_Cellular oxidant detoxification

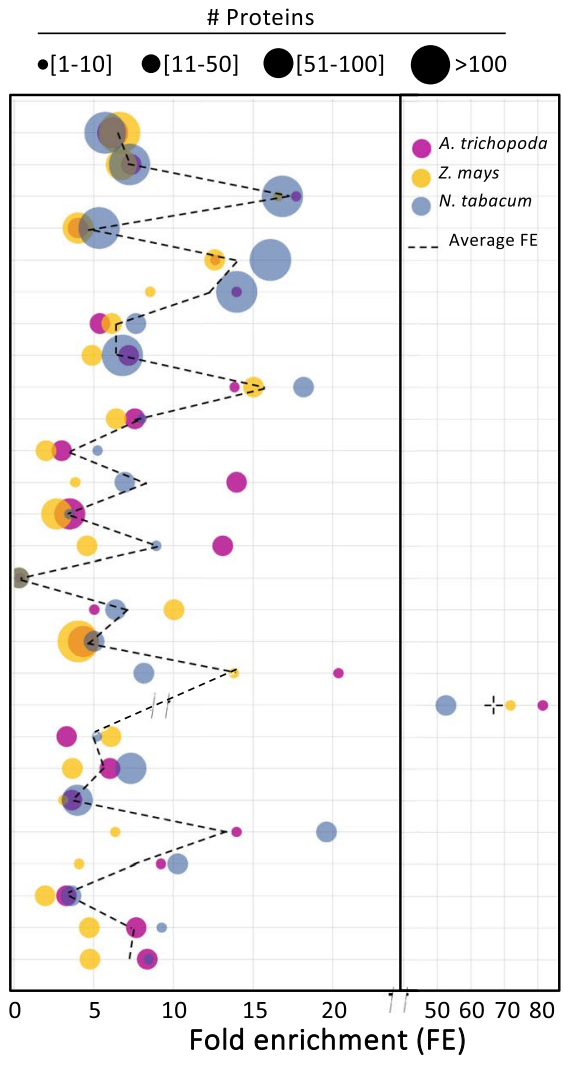

C

Protein description

Beta-galactosidase

Beta-glucosidase

Exo-polygalacturonase

Expansin

Extensin

Glucan endo-1,3-beta-glucosidase

Cell wall Glycosyl hydrolase domain related

Pectin acetylesterase

Pectin lyase-like protein

Pectinesterase

Phosphodiesterase

Pectin methylesterase inhibitor (PMEI)

Polygalacturonase

Rapid alkalinization factor (RALF)

UDP-arabinopyranose mutase UTP-G1-phosphate uridylyltransferase Fasciclin-like arabinogalactan

Cell surface GPI-LORELEI like (LLG)

Pollen allergen

Actin depolimerization factor

Cytoskeleton Profilin

Villin

6-phosphogluconate dehydrogenase

Aldolase

Enolase

Energy

Fructose-bisphosphate aldolase

metabolism

Glucose-6-phosphate isomerase

Malate dehydrogenase
A.t Z.m. N.t

$\begin{array}{lll}3 & 3 & 5 \\ 7 & 1 & 6\end{array}$

$\begin{array}{lll}7 & 1 \\ - & 4 & 1\end{array}$

-4
-16

16
$-\quad 31$

$\begin{array}{lll}6 & 3 & 7\end{array}$

$\begin{array}{lll}7 & 8 & -\end{array}$

$\begin{array}{lll}7 & 8 & - \\ - & 1 & 4\end{array}$

$\begin{array}{lll}1 & 9 & -\end{array}$

3920

2 - -

$\begin{array}{lcc}2 & - & - \\ 3 & 14 & 5\end{array}$

$\begin{array}{lll}3 & 1 & 1\end{array}$

1
-4

- 312

$\begin{array}{lll}1 & 3 & 2 \\ 4 & 6 & 7\end{array}$

1

2

$\begin{array}{lll}2 & 5 & 9 \\ - & 8 & 6 \\ 1 & 6 & 5\end{array}$

-

2

1
2

Phosphoglucomutase

Phosphoglycerate kinase

Triosephosphate isomerase

ATP synthase

\begin{tabular}{|c|c|c|c|c|}
\hline & Protein description & A.t & Z.m. & N.t \\
\hline \multirow{5}{*}{$\begin{array}{c}\text { Lipid } \\
\text { metabolism }\end{array}$} & Acyl carrier protein & 2 & - & 6 \\
\hline & Acyl-CoA-binding protein & 1 & 1 & 2 \\
\hline & GDSL esterase/lipase & 2 & 6 & - \\
\hline & Lipid transfer protein & 10 & 9 & 4 \\
\hline & Phospholipase & 1 & 1 & 4 \\
\hline \multirow{6}{*}{ Protection } & Chitin binding protein & 3 & - & - \\
\hline & Defensin-like & - & 2 & - \\
\hline & Glyoxalase & 2 & 3 & 5 \\
\hline & Heat shock protein & 3 & 2 & 22 \\
\hline & Late embryogenesis abundant & 4 & 1 & - \\
\hline & Glutathione S-transferase & 4 & 4 & 3 \\
\hline \multirow{3}{*}{ Proteolysis } & Cysteine proteinase & 1 & 3 & 3 \\
\hline & Proteasome & 2 & 8 & 42 \\
\hline & Subtilisin-like Ser endopeptidase & 3 & 5 & - \\
\hline \multirow{4}{*}{ Redox } & Peroxidase & 14 & 5 & 9 \\
\hline & Phytocyanin domain & 13 & 2 & - \\
\hline & SKU5 similar & - & 5 & - \\
\hline & Superoxide dismutase & 2 & 2 & 8 \\
\hline \multirow{4}{*}{ Signalling } & 14-3-3 domain & 2 & 3 & 8 \\
\hline & Calcium binding protein & 1 & 2 & 13 \\
\hline & Calmodulin & 3 & 2 & 5 \\
\hline & Translat. contr. tumor prot. (TCTP) & 1 & 1 & 5 \\
\hline \multirow{4}{*}{ Synthesis } & L-homocysteine biosynthesis & 1 & 1 & 4 \\
\hline & Nucleoside diphosphate kinases & 1 & 2 & 5 \\
\hline & Peptidyl-prolyl cis-trans isomerase & - & 6 & 13 \\
\hline & Ribosomal protein & 7 & - & - \\
\hline \multirow{5}{*}{$\begin{array}{l}\text { Vesicular } \\
\text { transport }\end{array}$} & Calreticulin & - & 2 & 2 \\
\hline & Coatomer & 2 & 6 & 4 \\
\hline & Golgi associtaed & 1 & 1 & - \\
\hline & Luminal binding protein & 2 & 2 & 6 \\
\hline & Protein disulfide-isomerase & 3 & 2 & 5 \\
\hline Unknown* & & 4 & 17 & 10 \\
\hline
\end{tabular}


4Fig. 3 GO term analysis of secreted proteins from A. trichopoda, Z. mays and N. tabacum. a Venn diagram representing in brackets the total number of significantly enriched GO terms for biological processes in each species and the overlapping number. b Overlapping GO terms of all three species, number of detected proteins in each category, their fold enrichment (FE) and mean value indicated by a dashed line. c Most relevant secreted proteins when comparing A. trichopoda (A.t.), Z. mays (Z.m.) and N. tabacum (N.t.) pollen secretomes. (*) No available information (see Supplemental Table S3)

strategy outlined in Supplemental Table S4a. In brief, we first searched in the current version of UniProtKB database and then in BLASTP and BLASTP for conserved protein domains. With this procedure, we could obtain already a description for many of these proteins. For those that remained uncharacterized, a BLASTP search was performed. In the case of maize and tobacco, a best-hit BLASTP search retrieved proteins exclusively described in other Poaceae or Solanaceae species, respectively (Supplemental Table S4c, d). In contrast, for Amborella the best-hit results were less specific, and they were found in many different plant species, ranging from other basal angiosperms to eudicots, monocots or even gymnosperms like Picea sitchensis (Supplemental Table S4b). Notably, at the end of this procedure there were only four uncharacterized proteins left in Amborella, 17 in maize and 10 in tobacco. These were described as "unknown" and labeled in yellow in Supplemental Table S4b, c, d.

Next, we investigated the proportion of proteins predicted to be secreted. We performed an in silico analysis to distinguish between the "conventional" and "unconventional" secretory pathway (see also Hafidh et al. 2016). As "conventional," we classified proteins that contain a signal peptide for secretion via the ER and Golgi. Proteins were categorized as "unconventional" if they were predicted to be secreted but lack a typical signal peptide and as "ambiguous" those that were unclear. Unconventionally secreted proteins were predicted by using the SecretomeP 2.0 online server that uses algorithms that recognize the presence of common motifs and sequences that have been previously found in many proteins secreted by non-classical paths.

Notably, as shown in Fig. 2b, the Amborella secretome showed equal preference for conventional (36.7\%) and unconventional secretion $(31.1 \%)$, respectively, whereas in the maize secretome the conventional secretion was prevalent (43.2\%; unconventional $28.6 \%$ ). In tobacco, the unconventional pathway (39.9\%) seemed to be expanded, while the conventional was reduced $(22.3 \%)$. In conclusion, these numbers indicated that we detected proper secretomes from all three species and that many proteins are secreted by unconventional pathways. However, the significantly different numbers of detected proteins and annotation problems with data from tobacco do not allow yet conclusions about evolutionary aspects of prevalence of secretory pathways among different angiosperms.

\section{Secreted pollen tube proteins are especially enriched in functions associated with the cell wall, signaling and energy metabolism}

To gain insights into biological functions of secreted proteins, a gene ontology (GO) terms enrichment analysis was performed by using PANTHER v15.0 (Supplemental Table S5) using the above-mentioned 335 proteins from Amborella, 552 from maize and 798 from tobacco. As shown in Fig. 3a, a total of 181 significantly enriched GO terms were found for the Amborella secretome, 305 for maize and 419 for tobacco, with an overlap of 105 between the three species. This indicated that $37 \%$ GO terms overlap between the basal angiosperm Amborella and the other two species, but only $26 \%$ in the maize and $20 \%$ of the tobacco secretomes, indicating a higher specificity of functions in more recently diverged sister clade angiosperms. However, many proteins occur in more than one GO term and the number of different GO terms increases with the number of proteins; thus, these conclusions should be taken with care. The enrichment of the main common GO terms and the number of proteins that are involved in each of them are represented in Fig. 3b. This figure showed that enriched biological processes related to energy metabolism, signaling, Golgi localization or the cell wall are on average equally represented in the three species, whereas processes related to reactive oxygen species (ROS), calcium-mediated signaling or unfolded protein response are enriched either specifically in Amborella or tobacco.

For a deeper analysis, the description of all proteins in the secretome data was associated with a biological function and the presence compared for each category. As shown in Fig. 3c, proteins associated with the cell wall, cell surface and signaling including vesicular transport, but also energy and lipid metabolism as well as proteolysis were present in the secretomes. With the exception of proteins associated with redox processes as well as ATP synthase components and ribosomal proteins, there was no significant enrichment of proteins in the Amborella secretome compared with the other species. The latter two protein groups hint at a minor contamination in the Amborella samples from burst pollen tubes, which could be associated with the fact that pollen grains had to be incubated for 14 hs to generate tubes of a significant length. Compared with the other two species, there is a remarkable enrichment of expansins, pectin methylesterase inhibitors (PMEIs), pectin lyases and rapid alkalinization factors (RALFs) in maize. Cell wall enzymes like pectinesterases and UDP-arabinopyranose mutases are enriched in tobacco, but also heat shock proteins and proteasome components. The latter two categories indicate also 


\section{A. trichopoda}

PG PT S Description

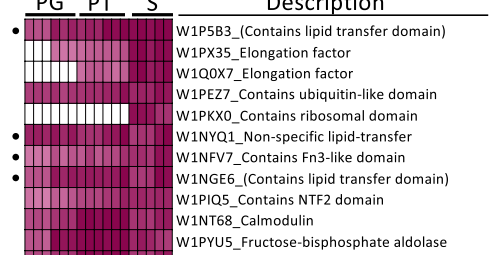

W1PYU5_Fructose-bisphosphate aldolase

WTPsL_Calmodulin

W1NHU3 (Contains glycosyl hydrolase domain)

U5CQU6 Calmodulin

U5CR20_Contains acyl-COA-binding domain

W1PWW3_(GPI-anchored protein)

W1PJM9_(Triosephosphate isomerase)

U5Dsm_ (Co shock protein 701

U5DFN7_Contains phytocyanin domain

W1NI38_Contains C2 domain

W1PMQ5_Non-specific lipid-transfer

U5DB66_Contains oxidoreductase domain

W1PGJ2_Acyl carrier protein

W1NZ92__Luminal binding protein)

W1PZE8 Contains phytocyanin domain)

W1NYZ2_Superoxide dismutase

W1NX86_Biotin carboxyl carrier protein

U5D853_(Thaumatin-like protein)

W1NUG2_Thioredoxin

U5DBW2_Phosphoglycerate kinase

W1NIU4_Contains C2 domai

Q70XZ6_ATP synthase

\begin{tabular}{l|l|l|l|l|l|l|l|l} 
W1NZ42_ATP synthase \\
W1PPZ8_(Cu-oxidase) \\
W1NE67_Contains phytocyanin domain
\end{tabular} W1NLZ5_Plastocyanin

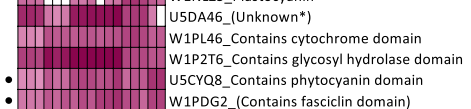

W1PDG2_(Contains fasciclin domain)

W1P161_Contains FAS1 domain

W1NLJ_Contains chitin-binding domain

W1PWH4_Contains lipid transfer dom

U5D444_(Glutathione S-transferase)

W1PCA7_Beta-galactosidase

W1NLF__(Contains LysM domain)

W1NHH8_(Hydrolase)

W1NIA7_(Luminal-binding protein)

W1NEY8_Contains ML domain

W1P3A1_Contains Barwin domain

W1PLT7_(Mitochondrial carrier domain)

U5DGC3_Contains FAS1 domain

W1PY03_Contains flavodoxin-like domain

W1PYO3_Contains flavodoxin-like domain
W1PZBO_Contains plastocyanin-like domain

W1NWW8_(Contains ATPases domain)

W1PD43_ATP synthase subunit be

W1NFC3_Contains PMEI domain

U5D3P0 Superoxide dismutase

U5D361 Acyl carrier protein

\begin{tabular}{l|l|l|l|l|l} 
USCVAO_Contains lipid transfer domain \\
W1PQEO_Ribulose-phosphate 3-epimerase
\end{tabular}

W1PGS3_Glycoside hydrolase

W1P8K1_Peroxidase

WINMG__Pectate lyase

W1PBA_Clonins cupin do

W1NUK2 Acetyltransferase

W1PSV6_Peroxidase

W1NLZ8_Fructose-bisphosphate aldolase

U5D7C1_(Endoribonuclease)

W1PTNO_Contains plastocyanin-like domain

W1PZZO_COS ribosomal protein S27

W1PQM1_Adenosylh

W1NPM5_Elongation factor

W1PRG__Glyceraldehyde 3P-dehydrogenase

\begin{tabular}{l||l|l|l|l|l} 
W1PRG7_Glyceraldehyde 3P-dety \\
U5CY76_(Phosphodiesterase) \\
W1NV71_Contains PMEI domain
\end{tabular}

U5D4L5_(Glyoxalase)

W1PAP7_Peroxidase

W1PIZ9-Glyceraldehyde 3P-dehydrogenase

W10049_(O-fucosyltransferase)

W1P8B5_(Transaldolase)

U5D4Z6_Peroxidas

W1PGQ1_Contains DLH domain

U5CYLO_Contains tRNA ligase domain

W1PAX2_Protein disulfide-isomerase

U5D2T1_(Pectin lyase)

W1PR56_Glyceraldehyde 3P-dehydrogenase

W1NLW5_Contains H-ATPase domain
Z. mays

PG PT $S$ Description

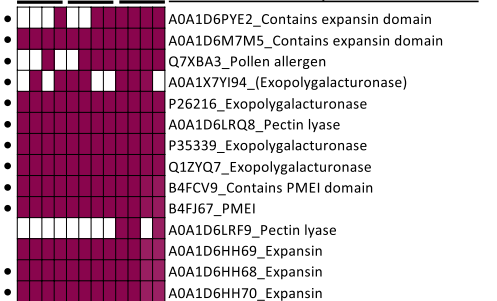

$\vdots$
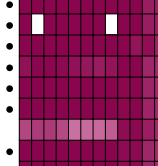

Q1zYQ8_Expansin

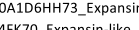

AOA1D6P228_FAD-binding

POC1Y5_Expansin

Q19K79_Beta expansi

COPSE1_Purple acid phosphatase

AOA1D6JNY2_Peroxidas

-
K7VQBO_Peroxidase
AOA1D6MK38_Lipid transfer-like
COPNL3_Profilin
K75C7_(Un

:

(

BGTVW3_(Unknown*)

AOA1R3LXF8 Glycosyl hydrolase

$: \quad$ B4FM79_Fasciclin-like

B6T6Y5_Profilin

A0A1D6HJ12_Uclacyanin

DA1D6IWU3_Purple acid phosphatas

4FCC1_Pollen-specific protein-like

A4KA55_Profilin

P35083_Profilin

B4FCPO_Profilin

B4FVG6_Pectin acetylesterase

A0A1D6MG24_Lipid transfer-like

B4F828_Pectin lyase-like

AOA1D6H438_Pectin lyase-like

A0A1D6H471_Pectin lyase-like

A0A1D6GU99_Beta-glucosidase

B4FZZ2_Peptidyl-prolyl isomerase

A0A1D6Q9F7_Uclacyanin

BAFDT7_(Contains PMEI domain)

K7VJE6_Putative subtilase

COP429_UTP-glucose-uridylyltransferase

COP2K2_SKU5 similar

COPFW1_SKU5 simila

A0A1D6M0X0_Pollen allergen Phl p 11

C4J531_(Unknown*)

A0A1D6PP81_Pollen extensin-like

P08440_Fructose-bisphosphate aldolase

B4G0K4_Phosphoglycerate kinase

protein

AOA1D6H4X3_(Dehydrogenase)

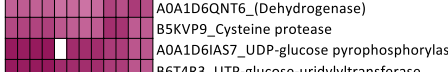

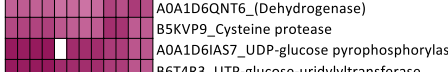

BTAR__UTP-glucose-uridyly|transferas

Q

- dehydrogenase

omutase

A0A1D6GJ73_Phosphoglucomut

(a)

BAFXT7_Expansin

58738_Expansin

AOA1D6HWN (Contains PMEI domain)

A0A1D6JFJ4_Pectinesterase

P12863_Triosephosphate isomerase

A0A1D600P1 Eukaryotic aspartyl protea

009054 Glyceraldehyde-3P- dehydrogenase

BGTRH9_Pollen allergen Phl p 2

B6T2Z8_Pollen allergen Phl $P 2$

COP439_UDP-arabinopyranose mutase

K7UUBO_Triose phosphate isomerase

BAFAL9_Fructose-bisphosphate aldolase

K7V794_Enolase

A0A1D6GVX2_Peptidyl-prolyl isomerase

A0A1D6GNG8_Nonspecific lipid-transfer

Q6VB92_Beta-glucanase

A0A1D6FSG9_SKU5 similar

B4FWPO_Fructose-bisphos

COHGV_Enolase

OA1D6H6F0_(Unknown*)

B46039_UDP-arabinopyranose mutase

6TEJ6_Fasciclin-like arabinogalacta

COHIO6_SKU5 similar

AOA1D6LQH9_Fasciclin-like

B4FQX1_Golgi associated protein
C4JAF6_Endo-1,3-beta-D-glucosidase
Protein abundance $\left(\log _{10} \mathrm{PA}\right)$

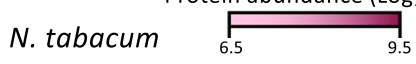

PT $\frac{\mathrm{S}}{\text { AOA1S4AZU4_Anther-specific protein LAT }}$ A0A1S4AZU4_Anther-specific protein LAT52
A0A153XV83_Anther-specific protein LAT52-like A0A154CB71_Anther-specific protein LAT52-like A0A154CB71_Anther-specific

A0A1S4D0K7_Ole
P41372_Profilin

P41372_Profilin
A0A1S4A3C7_Profilin

AOA1S4A3C7_Profilin
A0A1S3Z3L6_Profilin

A0A153Z3L6_Protilin

OA1S4DDY2_(Contains PMEI domain)

OA1S4D7F9 (Contains PMEI domain)

O1S3YHTG_Contains PMEI domain

ODA1S3ZHM9 Anther-specific protein LAT52-like

AOA154A8S3_Fasciclin-like

A0A153X462 UDP arabinopyanose mutase

A0A1S4BY21_Fasciclin-like

DA1S4CIX6_(Contains PMEI domain)

Fasciclin-like

DA1S4CCV4_Anther-specific protein LAT52-like

AOA1S4C365 UDP-arabinopyranose mutase 3

C5J0G6_Enolase

A1S3XTW2_Pectinesterase

A0A1S4ABZ9_Peptidyl-prolyl isomerase

AOA1S4BQ99_UDP-arabinopyranose mutase

A0A1S4BFD9_Peptidyl-prolyl isomerase

AOA1S4C2N5_UDP-arabinopyranose mutase

A0A1S3YD09_(Contains PAR1 domain)

AOA1S3ZMD5 UDP-arabinopyranose mutase

A0A1S3YCS2_UDP-arabinopyranose mutase

Q4G2Z0 Pectinesterase

AOA1S4B2B6_(Contains PAR1 domain)

AOA1S3YE50_Pectinesterase

AOA1S4A196_Peptidyl-prolyl isomerase

AOA1S3Z7T9_(Pollen allergen Ole e 6)

AOA1S4CWR8_Protein 108-like

A0A1S3ZZLO_Enolase-like

AOAS1S4BV28_Alcohol dehydrogenase-like

AOA1S3ZEE9_Glutelin-like

Q42953_Alcohol dehydrogenase

Q9FV64_Exopolygalacturonase-like

AOA1S4A8S1_Fasciclin-like

A0A1S4A2J8_Pectinesterase

AOA1S3Z6D5_GPI-anchored LORELEI-like

AOAS3XU16_Calcium-binding allergen Ole e 8-like

A0A1S4D817_Acyl-CoA-binding-like

Q76MF__Calmodulin

AOA1S4AWZ8_(Contains PAR1 domain)

AOA1S4CC96_GPI-anchored LORELEI-like

Q76ME6_Calmodulin

A0A1S4CPN9_Polygalacturonase-like

Q BVWY6_Ca+2-binding pollen allergen Nic 1

Q40454_Lipid transfer protein-like

AOAS4BCN3_Enolase

AOA1S4C3S3_L-ascorbate oxidas

P29162_L-ascorbate oxida

Q40567_Calretulin

Q42962_Phosphoglycerate kinas

nesis-related-like

Q76MF4_Calmodulin

Q6UN73_L-ascorbate oxidas

genase

AOA

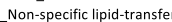

B5M6X8_Calreticulin

A0A153ZTX1 Superoxide dismutase

A0A1S3YXG6_Malate dehydrogenase

A0A1537828_Phosphoglycerate kinase

AOA1S4BN76_Endo-1,3-beta-glucosidase-like

A0A15325D7_Endo-1,3-beta-glucosidase-like

A0A1S3XQR5_Fructose-bisphosph

Q6IVK8 UDP-glucose dehydrogenas

A0A1S3ZW53 Malate dehydrogenase

Q9SDN7_Ole e 6-like

AOA1S4ATJ6 Steroid-binding

AOA1S4A6N1_Steroid-binding

Q6UN74_L-ascorbate oxidase

A0A1S3ZKZ4_Phosphoglycerate kinase

A0A1S3YZT7_Proteinase inhibitor-like

A0A1S3Z9J4_Pectinesterase

AOA1S4BAJ__Deaminase A-like

K7KNT9_Homocys-S-methyltransferase

M1D1L1_Acyl-CoA-binding

024330_Phosphoglycerate kinase

A0A1S3XXM3_Triosephosphate isomerase-like

A0A153XCV6_Homocys-S-methyltransferase

A0A154DNF2 Phosphoglycerate mutase-like

A0A075F1U0_Deaminase-like

AOA1S4BRT5_Beta-galactosidase

U3PLK3_Leucine aminopeptidase

AOA1S3XCW1_Pectinesterase

12FJN7_Polyadenylate-binding

AOA1S4C2F8_Polyadenylate-binding

AOA1S4CUG9_Endoglucosidase-like
A0A1S4B9P9_Pectinesterase 
४Fig. 4 Identity of top 100 most abundant pollen tube secreted proteins from A. trichopoda, Z. mays and N. tabacum. Each cell represents the $\log 10$-transformed protein area of a given protein accession in parts per million (ppm). White cells represent the absence of protein. Proteins were sorted according to the highest number in the secretome data on top. Features from uncharacterized proteins are given in brackets. (O) Proteins with predicted signal peptide. *Proteins without any available information. $P G$ pollen grain, $P T$ pollen tube, $S$ secretome

for tobacco a slight contamination with cytosolic proteins. In summary, similar biological processes were enriched in the secretomes of the three selected angiosperm species. Proteins enriched in maize or tobacco, respectively, points toward differences on pollen tube cell wall composition and pollen tube signaling.

\section{Majority of most abundant secreted proteins are either cell wall-modifying enzymes or small cysteine-rich proteins (CRPs)}

Next, we studied the identity of the top 100 most abundant proteins in the secretome samples, the presence of a signal peptide, and compared their abundance with that in pollen grain and pollen tube samples (Fig. 4). Protein abundance was calculated by the number of mapped peptides in each sample category for a given protein. Although this strategy discriminates against smaller proteins, we found that especially a number of smaller proteins including candidate signaling proteins were very abundant. Among the most often detected proteins in the Amborella samples are a larger number of different lipid-binding/transfer proteins (LTPs), elongation factors and a protein containing a ribosomal protein domain. As reported already above, especially different expansins are highly abundant in the secretome of maize, but also polygalacturonases, pectin lyases, peroxidases and small cysteine-rich proteins like LTPs, PMEI and pollen allergen Ole el confirming the data shown in Fig. 3c. Small cysteine-rich proteins representing also candidate signaling proteins like LAT52, pollen allergen Ole e1/6, PMEI and LTPs belong also to the most abundant proteins in the tobacco secretome. The second most abundant category is proteins involved in cell wall modification like polygalacturonases, UDP-arabinopyranose mutases and pectinesterases.

An in silico analysis revealed that $33 \%, 63 \%$ and $45 \%$ of the top 100 Amborella, maize and tobacco proteins contained a signal peptide. This number is similar to the number of all proteins in Amborella (see above), but significantly higher compared with the numbers for the conventional secretory pathway reported above for all maize and tobacco proteins, respectively. Notably, while most proteins were detected in all sample categories, proteins exclusively detected in the secretome of Amborella are above-mentioned protein containing a ribosomal protein domain, proteins with an SPX domain and tRNA ligase domain, respectively, as well as the cell wall enzymes $\mathrm{O}$-fucosyltransferase and pectin lyase. The latter was also exclusively detected in the secretome of maize in addition to an expansin, a gibberellic acid-regulated protein, a cysteine proteinase and small unknown proteins. Abundant proteins present in the secretome, but not in the pollen tube samples of tobacco, included cell wall enzymes like UDP-arabinopyranose mutase and pectinesterase, but also small CRPs like pollen allergens and LTPs as well as two GPI-anchored LORELEI-like proteins, whose homologs were also found in the Amborella secretome.

Finally, we wanted to explore the correlation between high transcript levels with abundancy of secreted proteins. Transcriptomic data for pollen grains and pollen tubes as well as vegetative control tissues were available in the CoNekT database (https://evorepro.sbs.ntu.edu.sg) for Amborella and maize. Similar data were not available for tobacco, and we thus restricted our final analyses on these two species. We selected all secreted proteins whose transcript levels were specifically expressed in male gametophytes with values of at least 100 transcripts per million (TPM) in pollen tubes of Amborella (Fig. 5a) and 1000 TPM in maize (Fig. 5b), respectively. Proteins were ordered in a manner that the strongest expressed genes were shown at the top. Additionally, we further classified proteins according to the presence or absence of a signal peptide. We observed that in Amborella there was an equal number of highly expressed genes encoding proteins with a signal peptide (16 proteins) and without (17 proteins). In maize, this number was approximately four times higher for genes encoding proteins with a signal peptide (55 proteins) compared with those lacking a signal peptide (15 proteins). This analysis detected the majority of the top 100 most abundant proteins (Fig. 4) indicating a strong correlation between transcriptomic and proteomic data. A few strongly expressed genes encoding, for example, a GPI-anchored LORELEI-like protein or LTPs in Amborella as well as a few unknown proteins in maize were only detected in few protein samples indicating low translation and high protein turnover rates, respectively. Notably, in the categories with a predicted signal peptide, almost all strongly expressed genes in both species encode either (i) CRPs like PMEIs, LTPs, RALFs, pollen allergens, defensin-likes and others or (ii) cell wall-modifying enzymes like galactosidases, glucanases, polygalacturonases, pectinesterases, pectate lyases, expansins and peroxidases as well as (iii) a few small proteins of unknown functions. The latter category is especially interesting as it includes with proteins A0A1D6GCK9, K7V5C7, B6T2I1, B6TVW3, A0A1D6KI02, A0A1D6EGH6 and A0A1D6I6M4 small novel secreted proteins that are described here for the first time and which are almost exclusively enriched in the secretome. As shown in Fig. 5c, K7V5C7, for example, encodes a predicted mature peptide of 42 amino acids 


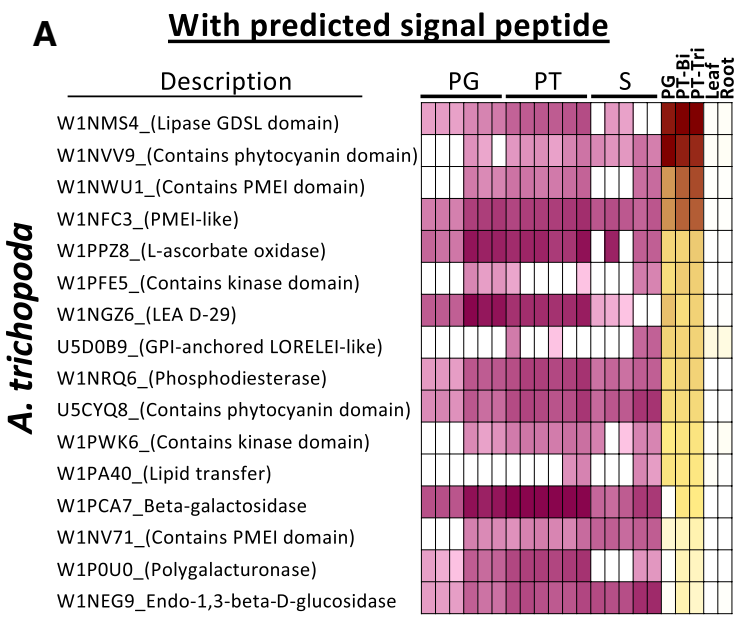

B

\section{Description}

B4FKQ2_Pollen-specific protein-like A0A1D6HWB9_ZmRALF5 A0A1D6GCK9_(Unknown*) COHI06_SKU5 similar A0A1D6FSG9_SKU5 similar A0A1D6GVX2_Peptidyl-isomerase B6T249 Cysteine-rich protein B6UE55_ZMRALF2 BGTULO_ZmRALF3 K7V5C7_(Unknown*) B4FMP9 (Contains PMEI domain) P26216 Exopolygalacturonase B4FPA9_Expansin B4FCY2_Pectinesterase A0A1D6KM59_Expansin A0A1D6KM60_Expansin B6T2I1_(Unknown*) B4FCV9_(Contains PMEI domain) B6TVW3_(Unknown*) B6TGEO_Pectinesterase inhibitor-like A0A1D6M0U7_(Contains PMEl domain) B4FJ67 Lustrin A

Q7XBA3_Pollen allergen

气. B4FWR8_Expansin

б. B4FMX__(Unknown*)

A0A1D6IWD7 LRR-family protein

N BAFBU1_Pollen allergen Cyn d 23 B4FM79_Fasciclin-like A0A1D6HJV5_Peroxidase A0A1D6HM43_Endoglucanase K7V1E7 Peroxidase B4FDT7_(Contains PMEI domain) B4FCC1_Pollen-specific protein-like A0A1D6P2H6_Pectate lyase B4FPD6_Alpha carbonic anhydrase K7WCD1_Expansin B4FXT7 Expansin

A0A1D6H4M3_Defensin-like B4FK70_Expnasin-like B4FLF1_Contains PMEI domain P58738 Expansin

A0A1D6MV60_Pathog.-related protein K7V4R8_Beta-galactosidase A0A1D6JNY2_Peroxidase A0A1D6MEIO_Pathog.-related protein A0A1D6KI02_(Unknown*) A0A1D6I6M4_(Unknown*) A0A1D6PP81_Pollen extensin-like COP2K2_SKU5 similar COPFW1_SKU5 similar B4F828 Pectin lyase-like AOA1D6EET4_Defensin-like AOA1D6EGH6_(Unknown*) C4JAF6_Endo-1,3-beta-D-glucosidase

\section{Without predicted signal peptide}
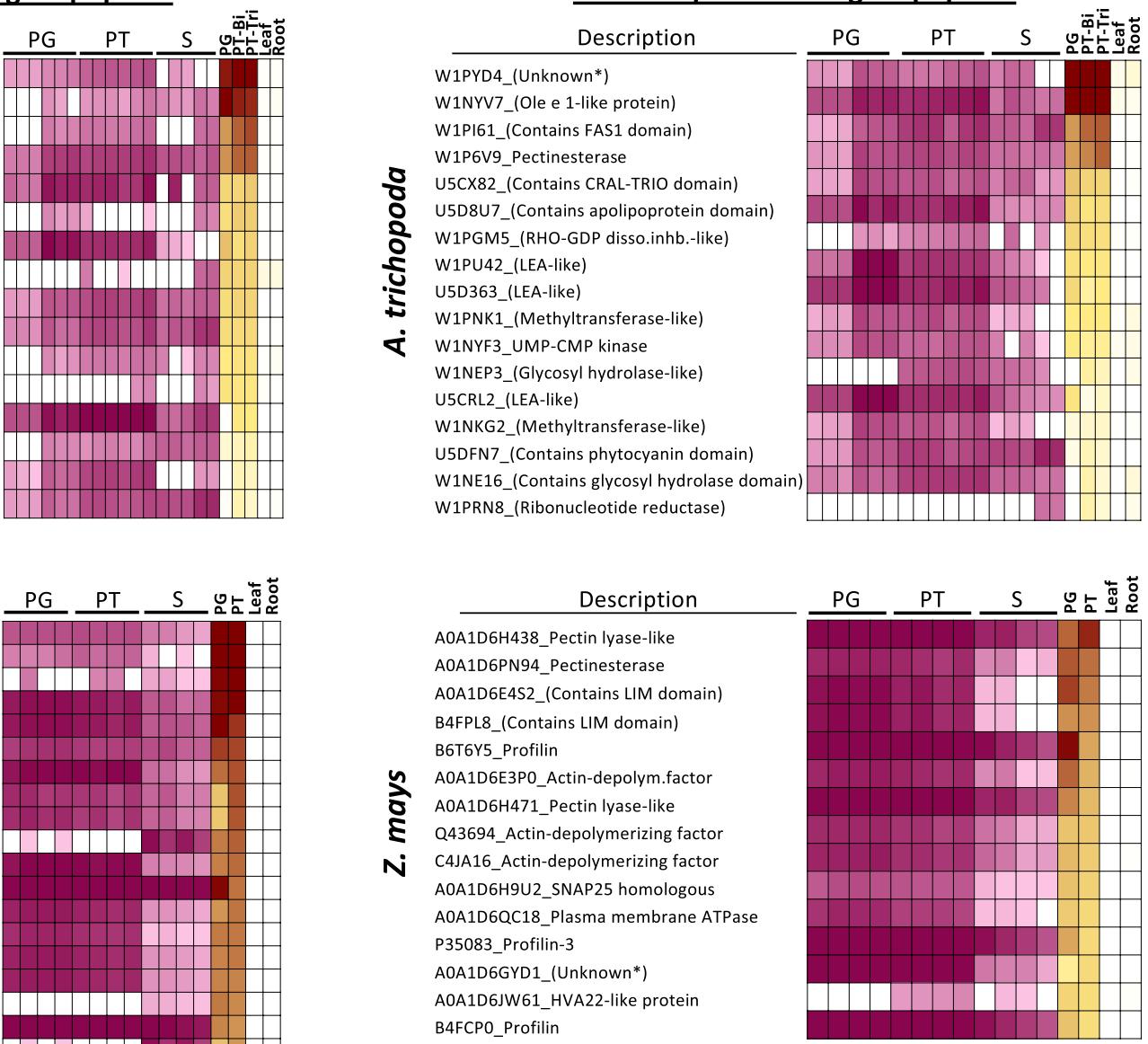

Protein abundance ( $\log _{10}$ protein area) Transcript level (TPM)

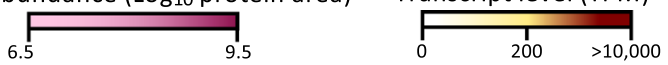

C

\section{B6TULO (ZmRALF3)}

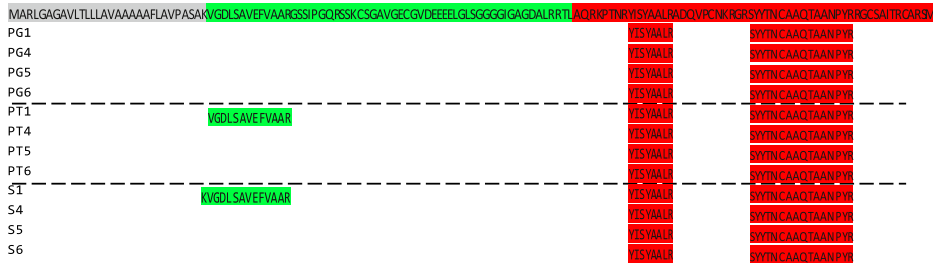

K7V5C7 (Unknown)

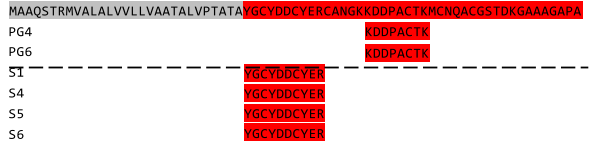

B6T2I1 (Unknown)

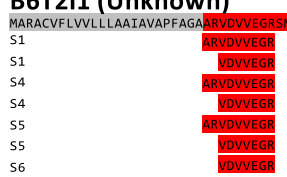


४Fig. 5 Comparison of proteomic and transcriptomic heatmaps of top expressed genes in pollen grains and pollen tubes of A. trichopoda and Z. mays as well as examples of mapped peptides. Genes and corresponding proteins were sorted with those showing the highest transcript levels on top. Two vegetative tissues are also included. $\mathbf{a}$ and $\mathbf{b}$ Proteins whose genes display at least 100 TPM expression levels in pollen tubes at the tricellular stage of A. trichopoda and 1000 TPM in pollen tubes of $Z$. mays are shown. Left blocks display proteins with predicted signal peptides and right blocks those lacking a predicted signal peptide. Color code for protein abundance and transcript level is indicated. Each biological protein sample is indicated. Average transcript levels were taken from Flores-Tornero et al. (2020). White cells represent the absence of protein or transcript. Features from uncharacterized proteins are given in brackets, and asterisks mark proteins without any available information. c Three examples showing mapping of identified peptides to small secreted proteins. $P G$ pollen grain, $P T$ pollen tube, $S$ secretome

likely stabilized by 6 cysteines, while B6T2I1 generates a predicted secreted peptide of 48 amino acids that might be further processed as it lacks cysteines and we only detected peptides of 7 and 9 amino acids length, respectively, after the predicted $\mathrm{N}$-terminal cleavage site. For other secreted proteins like RALF3 (Fig. 5c), we detected pro-protein peptides in the secretome, indicating that processing to fully mature proteins occurs (also) in the apoplast. In summary, the majority of most abundant secreted proteins or most strongly expressed genes encoding secreted proteins are either cell wall-modifying enzymes or CRPs. Finally, we detected novel secreted proteins and showed how these data can be used to study also processing of secreted proteins.

\section{Discussion}

\section{Enrichment of cell wall enzymes and CRPs in pollen tube secretomes}

The observation that we detected especially cell wall enzymes and CRPs in pollen tube secretomes was not surprising taking into consideration that these belong also to the strongly expressed genes (e.g., Bokvaj et al. 2015; Conze et al. 2017; Tan et al. 2018). Moreover, pollen tubes are fast growing plant cells that require a solid cell wall and that communicate intensively with the surrounding maternal tissues. Surprisingly, we also found many enzymes involved in lipid and energy metabolism, but also in proteolysis and redox processes as well as predicted cytoplasmic proteins like cytoskeletal components and heat shock proteins. These observations point toward the possibility that the secretomes might have been contaminated with cytoplasmic proteins from burst pollen tubes. However, $>70 \%$ identified proteins were predicted to be secreted; the composition of the secretomes was highly different compared with that from pollen tubes in each species and very homogenous in itself in independent experiments as shown by PCA analyses. Moreover, gene ontology terms associated with the above processes were significantly overrepresented in the secretomes of all three species compared with other tissues, indicating that contaminations were negligible.

During their journey, pollen tubes require many nutrients from the style, a lot of energy, but also structural components to generate cell wall material and membranes. Thus, it is not unlikely that many enzymes and structural components were secreted to contribute to the high speed of pollen tube growth. Notably, a similar observation has also been reported recently in studies about reproductive secretions in gymnosperms (Prior et al. 2019): Predicted intracellular and cytoplasmic proteins were detected in pollination droplets of naked ovules. The biological relevance of these proteins in the extracellular space is not clear. Moreover, another study about pollen tube secretions in Olea europaea not only described the presence of GAPDH, fructokinases and actin in the secretome, but also pointed toward the relevance of the unconventional secretory pathway to secrete cytosolic proteins (Prado et al. 2014). To which extent the in vitro obtained secretome data reflect the in vivo secretomes at different stages of the pollen tube journey is also unclear. It has been shown that germination media influence the pollen tube transcriptome in Arabidopsis thaliana (da Costa et al. 2013) and thus may also have an impact on the secretome. Moreover, in the same species it was shown that the pollen tube transcriptome also changes during growth through the stigma and style (Qin et al. 2009) and thus likely also leads to changes in the secretome composition. So far, differences in the secretome from pollen tubes germinated in vitro and semi-in vivo have only been reported in tobacco (Hafidh et al. 2016) and were discussed to occur due to the intensive cross talk between pollen tubes and female tissues during stigma-style penetration and growth (Dresselhaus and Franklin-Tong 2013) that is lacking during pollen tube growth in vitro.

\section{Correlation of secreted proteins with pollen tube growth rate and defense responses}

Notably, compared with Amborella, significantly more enzymes required for cell wall synthesis and modification as well as energy metabolism were detected in the maize and tobacco secretomes, explaining that their pollen tubes are capable to grow much faster. Additionally, this may also reflect the length of the journey: While pollen tubes in maize travel up to $30 \mathrm{~cm}$ (Zhou et al. 2017), tubes of tobacco grow around $4.5 \mathrm{~cm}$ (Cheung et al. 2000) and those of Amborella only about $0.12 \mathrm{~cm}$ (Williams 2009). These findings may also reflect differences in cell wall compositions, thickness and elasticity among the three species. For instance, the presence of many expansins in maize involved in cell wall loosening likely reflects the necessity of elastic walls 
during high-speed tube growth as cell walls of grasses also contain low amounts of pectins and xyloglucans compared with other angiosperms (Cosgrove 2015). The low number of beta-glucosidases detected in the maize secretome correlates with the low number of genes encoding for beta-glucosidases in this species compared to others (Gomez-Anduro et al. 2011). PMEIs that regulate cell wall elasticity (Wormit and Usadel 2018) are abundantly transcribed and translated in all three species investigated. However, 35 of 49 PMEIs were reported to be specifically transcribed and translated in pollen grains of maize (Zhang et al. 2019). Altogether, this suggests that the wall of fast-growing maize pollen tubes remains very elastic during growth, but hints also to the possibility that cell walls of maternal tissues have to be loosened at high speed to allow pollen tube penetration. Tobacco secretes abundantly UDP-arabinopyranose mutase, which correlates with findings in other tissues, showing that arabinoxyloglucans are especially abundant in cell walls of solanaceous plants (Honta et al. 2018).

In addition to proteins involved in energy and lipid metabolism as well as cell wall synthesis and modification, many CRPs were detected in the secretomes. Allergens like Pollen Ole e 1 have been abundantly detected in maize and tobacco. They have also been reported previously in in vitro pollen tube secretions from Olea europaea and were suggested to play a possible role in signaling (Alché et al. 2004). LTPs, which are considered as key proteins in lipid barrier polymer synthesis and extracellular signaling in many tissues (Salminen et al. 2016), were also shown as components regulating pollen tube adhesion to the stigma and style in Lilium longiflorum (Park et al. 2000) and were detected in all three species. Genes encoding RALFs that have been shown to regulate pollen tube growth and cell wall integrity are massively and specifically transcribed in both male and female gametophytes of all angiosperm clades (Campbell and Turner 2017; Flores-Tornero et al. 2019, 2020; Ge et al. 2017; Mecchia et al. 2017). Although we detected RALFs in the maize secretome, they were not among the top 100 most abundantly secreted proteins and some were only found in the pollen grain and pollen tube fractions, respectively. Similarly in Amborella, where members of the RALF family belong to the highly expressed genes in male gametophytes (Flores-Tornero et al. 2020), proteins were detected only in pollen grain and pollen tube protein extractions, respectively. In Arabidopsis thaliana, RALF4/19 have been characterized as small peptides that are highly transcribed and abundantly secreted from pollen tubes (Ge et al. 2017; Mecchia et al. 2017). It could thus be expected that their homologs in the eudicot tobacco are also abundant in the secretome, which is not the case. This finding points toward the possibility that RALF peptides are less stable in the extracellular space compared with LTPs, Pollen Ole e $1 \mathrm{~s}$ and other larger CRPs. The presence of cysteine proteinases, subtilisin-like serine endopeptidase and other aminopeptidases in pollen tube secretomes supports the hypothesis that some proteins are rapidly degraded, while others are more stable.

Proteins related to protection like chitin binding proteins, thaumatins, LEA proteins or proteins containing Barwin or ML domains are among the top 100 most abundant proteins in the Amborella, but not in the maize and tobacco secretomes, respectively. Notably, their homologs were also present in pollination drops from gymnosperms (Prior et al. 2019). This observation hints toward the hypothesis that wild plant species spend more efforts and energy into defense and thus contain higher amounts of these proteins in their apoplasts, while cultivated species like maize and tobacco are bred for high yield in sacrifice for the generation of protection proteins.

\section{Conclusions and outlook}

In conclusion, we provide here a simple and very robust procedure that could potentially be used for many other angiosperms to obtain pollen tube secretomes. The analysis of the secretome from three phylogenetically distant angiosperm clades shows that-similar to other plant tissues (Krause et al. 2013) —on average both, conventional and unconventional secretory pathways are used at similar rates to secrete proteins from growing pollen tubes to the apoplast. Identified cell wall enzymes differ in abundance significantly among the three plant species analyzed likely reflecting differences in cell wall types and their elasticity in response to varying pollen tube growth speed and length. Novel small secreted proteins or peptides were discovered in secretomes and partially unprocessed pro-peptides pointing toward final cleavage steps in the apoplast, which is rich in proteases. For future studies, we now recommend to extract higher protein amounts and generate more mapped peptides after LC MS/MS analysis as the data generated are very useful to elucidate also the ultimate sequence and posttranslational modification(s) of secreted mature proteins. Improvements in LC MS/MS technology may detect more and less abundant proteins in pollen tube secretomes in future studies, but we think that the data presented provide already a valuable resource for gene/protein discovery for functional studies as well as for comparative studies involving further angiosperm species to better understand the evolution, function and specificity of pollen tubes and their journey.

Author contributions statement FV initiated the study. SS and TD designed the study. MFT and LW generated the samples, $\mathrm{SH}$ and DH performed the analyses, DP and ZZ performed the proteomic analyses, and MFT analyzed the data and wrote the manuscript with TD. 
Acknowledgements We are grateful to Maximilian Weigend, Cornelia Löhne and Bernhard Reinken (Botanical Garden of the University of Bonn, Germany) for providing Amborella trichopoda plant material. We acknowledge the support of MEYS CR via the project "Centre for Experimental Plant Biology" (CZ.02.1.01/0.0/0.0/16_019/00007 38 ) and the CIISB infrastructure project (LM2018127) for measurements at the CEITEC Proteomics Core Facility. Finally, we thank the German Research Council DFG for financial support via ERA-CAPS grant EVOREPRO (DR 334/12-1 to SS and TD) and a grant within the Collaborative Research Center SFB924 (to TD).

Funding Open Access funding enabled and organized by Projekt DEAL.

\section{Compliance with ethical standards}

Conflict of interest There is no conflict of interests.

Open Access This article is licensed under a Creative Commons Attribution 4.0 International License, which permits use, sharing, adaptation, distribution and reproduction in any medium or format, as long as you give appropriate credit to the original author(s) and the source, provide a link to the Creative Commons licence, and indicate if changes were made. The images or other third party material in this article are included in the article's Creative Commons licence, unless indicated otherwise in a credit line to the material. If material is not included in the article's Creative Commons licence and your intended use is not permitted by statutory regulation or exceeds the permitted use, you will need to obtain permission directly from the copyright holder. To view a copy of this licence, visit http://creativecommons.org/licenses/by/4.0/.

\section{References}

Agrawal GK, Jwa NS, Lebrun MH, Job D, Rakwal R (2010) Plant secretome: unlocking secrets of the secreted proteins. Proteomics 10:799-827. https://doi.org/10.1002/pmic.200900514

Alché JdD, M'rani-Alaoui M, Castro AJ, Rodríguez-García MI (2004) Ole e 1, the major allergen from olive (Olea europaea L.) pollen, increases its expression and is released to the culture medium during in vitro germination. Plant Cell Physiol 45:1149-1157. https ://doi.org/10.1093/pcp/pch127

Bircheneder S, Dresselhaus T (2016) Why cellular communication during plant reproduction is particularly mediated by CRP signalling. J Exp Bot 67:4849-4861. https://doi.org/10.1093/jxb/erw271

Bokvaj P, Hafidh S, Honys D (2015) Transcriptome profiling of male gametophyte development in Nicotiana tabacum. Genom Data 3:106-111. https://doi.org/10.1016/j.gdata.2014.12.002

Campbell L, Turner SR (2017) A comprehensive analysis of RALF proteins in green plants suggests there are two distinct functional groups. Front Plant Sci 8:37. https://doi.org/10.3389/ fpls.2017.00037

Chae K, Lord EM (2011) Pollen tube growth and guidance: roles of small secreted proteins. Annals Bot 108:627-636. https://doi. org/10.1093/aob/mcr015

Chae K, Gonong BJ, Kim S-C, Kieslich CA, Morikis D, Balasubramanian S, Lord EM (2010) A multifaceted study of stigma/style cysteine-rich adhesin (SCA)-like Arabidopsis lipid transfer proteins (LTPs) suggests diversified roles for these LTPs in plant growth and reproduction. J Exp Bot 61:4277-4290. https://doi. org/10.1093/jxb/erq228
Cheung AY et al (2000) Pollen-pistil interactions in Nicotiana tabacum. Annals Bot 85:29-37. https://doi.org/10.1006/ anbo.1999.1016

Chevallet M, Luche S, Rabilloud T (2006) Silver staining of proteins in polyacrylamide gels. Nat Protoc 1:1852-1858. https://doi. org/10.1038/nprot.2006.288

Conze LL, Berlin S, Le Bail A, Kost B (2017) Transcriptome profiling of tobacco (Nicotiana tabacum) pollen and pollen tubes. BMC Genomics 18:581. https://doi.org/10.1186/s12864-017-3972-3

Cosgrove DJ (2015) Plant expansins: diversity and interactions with plant cell walls. Curr Opin Plant Biol 25:162-172. https://doi. org/10.1016/j.pbi.2015.05.014

da Costa ML, Pereira LG, Coimbra S (2013) Growth media induces variation in cell wall associated gene expression in Arabidopsis thaliana pollen tube. Plants 2:429-440. https://doi.org/10.3390/ plants2030429

Dehors J, Mareck A, Kiefer-Meyer MC, Menu-Bouaouiche L, Lehner A, Mollet JC (2019) Evolution of cell wall polymers in tip-growing land plant gametophytes: composition, distribution, functional aspects and their remodeling. Front Plant Sci 10:441. https://doi. org/10.3389/fpls.2019.00441

Dresselhaus T, Franklin-Tong N (2013) Male-female crosstalk during pollen germination, tube growth and guidance, and double fertilization. Mol Plant 6:1018-1036. https://doi.org/10.1093/mp/sst061

Dresselhaus T, Sprunck S, Wessel GM (2016) Fertilization mechanisms in flowering plants. Curr Biol 26:R125-139. https://doi. org/10.1016/j.cub.2015.12.032

Flores-Tornero M, Proost S, Mutwil M, Scutt CP, Dresselhaus T, Sprunck S (2019) Transcriptomics of manually isolated Amborella trichopoda egg apparatus cells. Plant Reprod 32:15-27. https:// doi.org/10.1007/s00497-019-00361-0

Flores-Tornero M et al (2020) Transcriptomic and proteomic insights into Amborella trichopoda male gametophyte functions. Plant Physiol. https://doi.org/10.1104/pp.20.00837

$\mathrm{Ge} \mathrm{Z}$ et al (2017) Arabidopsis pollen tube integrity and sperm release are regulated by RALF-mediated signaling. Science 358:15961600. https://doi.org/10.1126/science.aao3642

Gomez-Anduro G, Ceniceros-Ojeda EA, Casados-Vazquez LE, Bencivenni C, Sierra-Beltran A, Murillo-Amador B, Tiessen A (2011) Genome-wide analysis of the beta-glucosidase gene family in maize (Zea mays L. var B73). Plant Mol Biol 77:159-183. https ://doi.org/10.1007/s11103-011-9800-2

Hafidh S, Potesil D, Fila J, Capkova V, Zdrahal Z, Honys D (2016) Quantitative proteomics of the tobacco pollen tube secretome identifies novel pollen tube guidance proteins important for fertilization. Genome Biol 17:81. https://doi.org/10.1186/s1305 9-016-0928-x

Honta H, Inamura T, Konishi T, Satoh S, Iwai H (2018) UDP-arabinopyranose mutase gene expressions are required for the biosynthesis of the arabinose side chain of both pectin and arabinoxyloglucan, and normal leaf expansion in Nicotiana tabacum. J Plant Res 131:307-317. https://doi.org/10.1007/s10265-017-0985-6

Huang Q, Dresselhaus T, Gu H, Qu LJ (2015) Active role of small peptides in arabidopsis reproduction: expression evidence. J Integr Plant Biol 57:518-521. https://doi.org/10.1111/jipb.12356

Johnson MA, Harper JF, Palanivelu R (2019) A fruitful journey: pollen tube navigation from germination to fertilization. Annu Rev Plant Biol 70:809-837. https://doi.org/10.1146/annurev-arplant-05071 8-100133

Krause C, Richter S, Knoll C, Jürgens G (2013) Plant secretome - from cellular process to biological activity. Biochimica Biophysica Acta 1834:2429-2441. https://doi.org/10.1016/j.bbapap.2013.03.024

Maruyama D, Higashiyama T (2016) The end of temptation: the elimination of persistent synergid cell identity. Curr Opin Plant Biol 34:122-126. https://doi.org/10.1016/j.pbi.2016.10.011 
Mecchia MA et al (2017) RALF4/19 peptides interact with LRX proteins to control pollen tube growth in Arabidopsis. Science 358:1600. https://doi.org/10.1126/science.aao5467

Mollet JC, Leroux C, Dardelle F, Lehner A (2013) Cell wall composition, biosynthesis and remodeling during pollen tube growth. Plants 2:107-147. https://doi.org/10.3390/plants2010107

Muschietti J, Dircks L, Vancanneyt G, McCormick S (1994) LAT52 protein is essential for tomato pollen development: pollen expressing antisense LAT52 RNA hydrates and germinates abnormally and cannot achieve fertilization. Plant $\mathbf{J}$ 6:321-338. https://doi. org/10.1046/j.1365-313X.1994.06030321.X

Park SY, Jauh GY, Mollet JC, Eckard KJ, Nothnagel EA, Walling LL, Lord EM (2000) A lipid transfer-like protein is necessary for lily pollen tube adhesion to an in vitro stylar matrix. Plant Cell 12:151-164. https://doi.org/10.1105/tpc.12.1.151

Prado N, Alche Jde D, Casado-Vela J, Mas S, Villalba M, Rodriguez R, Batanero E (2014) Nanovesicles are secreted during pollen germination and pollen tube growth: a possible role in fertilization. Mol Plant 7:573-577. https://doi.org/10.1093/mp/sst153

Prior $\mathrm{N}$ et al (2019) Complex reproductive secretions occur in all extant gymnosperm lineages: a proteomic survey of gymnosperm pollination drops. Plant Reprod 32:153-166. https://doi.org/10.1007/ s00497-018-0348-z

Qin Y et al (2009) Penetration of the stigma and style elicits a novel transcriptome in pollen tubes, pointing to genes critical for growth in a pistil. PLoS Genet 5:e1000621. https://doi.org/10.1371/journ al.pgen.1000621

Qu LJ, Li L, Lan Z, Dresselhaus T (2015) Peptide signalling during the pollen tube journey and double fertilization. J Exp Bot 66:51395150. https://doi.org/10.1093/jxb/erv275

Salminen TA, Blomqvist K, Edqvist J (2016) Lipid transfer proteins: classification, nomenclature, structure, and function. Planta 244:971-997. https://doi.org/10.1007/s00425-016-2585-4
Tan H, Zhang J, Qi X, Ye W, Wang X, Xiang X (2018) Integrated metabolite profiling and transcriptome analysis reveals a dynamic metabolic exchange between pollen tubes and the style during fertilization of Brassica napus. Plant Mol Biol 97:325-335. https ://doi.org/10.1007/s11103-018-0740-y

Williams JH (2009) Amborella trichopoda (Amborellaceae) and the evolutionary developmental origins of the angiosperm progamic phase. Am J Bot 96:144-165. https://doi.org/10.3732/ajb.0800070

Wormit A, Usadel B (2018) The multifaceted role of pectin methylesterase inhibitors (PMEIs). Intern J Mol Sci. https://doi. org/10.3390/ijms19102878

Zhang J et al (2017) Sperm cells are passive cargo of the pollen tube in plant fertilization. Nat Plants 3:17079. https://doi.org/10.1038/ nplants.2017.79

Zhang P, Wang H, Qin X, Chen K, Zhao J, Zhao Y, Yue B (2019) Genome-wide identification, phylogeny and expression analysis of the PME and PMEI gene families in maize. Sci Rep 9:19918. https://doi.org/10.1038/s41598-019-56254-9

Zhou LZ, Dresselhaus T (2019) Friend or foe: signaling mechanisms during double fertilization in flowering seed plants. Curr Top Dev Biol 131:453-496. https://doi.org/10.1016/bs.ctdb.2018.11.013

Zhou LZ, Juranic M, Dresselhaus T (2017) Germline development and fertilization mechanisms in maize. Mol Plant 10:389-401. https:// doi.org/10.1016/j.molp.2017.01.012

Publisher's Note Springer Nature remains neutral with regard to jurisdictional claims in published maps and institutional affiliations.

\section{Affiliations}

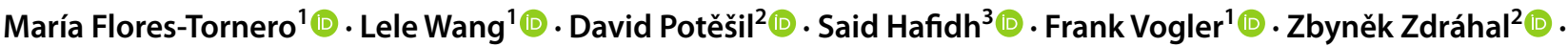 David Honys $^{3}{ }^{[0} \cdot$ Stefanie Sprunck ${ }^{1}\left[\right.$ Thomas Dresselhaus $^{1}{ }^{1}$}

1 Cell Biology and Plant Biochemistry, University of Regensburg, Universitätsstraße 31, 93053 Regensburg, Germany

2 Mendel Centre for Plant Genomics and Proteomics, Central European Institute of Technology, Masaryk University, Kamenice 5, 62500 Brno, Czech Republic
3 Laboratory of Pollen Biology, Institute of Experimental Botany ASCR, Rozvojová 263, 16502 Prague 6, Czech Republic 